\title{
Patterning of high mobility electron gases at complex oxide interfaces
}

Trier, Felix; Prawiroatmodjo, G. E. D. K. ; von Soosten, Merlin; Christensen, Dennis Valbjørn; Jespersen, T. S.; Chen, Yunzhong; Pryds, Nini

Published in:

Applied Physics Letters

Link to article, DOI:

$10.1063 / 1.4935553$

Publication date:

2015

Document Version

Publisher's PDF, also known as Version of record

Link back to DTU Orbit

Citation (APA):

Trier, F., Prawiroatmodjo, G. E. D. K., von Soosten, M., Christensen, D. V., Jespersen, T. S., Chen, Y., \& Pryds, N. (2015). Patterning of high mobility electron gases at complex oxide interfaces. Applied Physics Letters, 107, [191604]. https://doi.org/10.1063/1.4935553

\section{General rights}

Copyright and moral rights for the publications made accessible in the public portal are retained by the authors and/or other copyright owners and it is a condition of accessing publications that users recognise and abide by the legal requirements associated with these rights.

- Users may download and print one copy of any publication from the public portal for the purpose of private study or research.

- You may not further distribute the material or use it for any profit-making activity or commercial gain

- You may freely distribute the URL identifying the publication in the public portal 


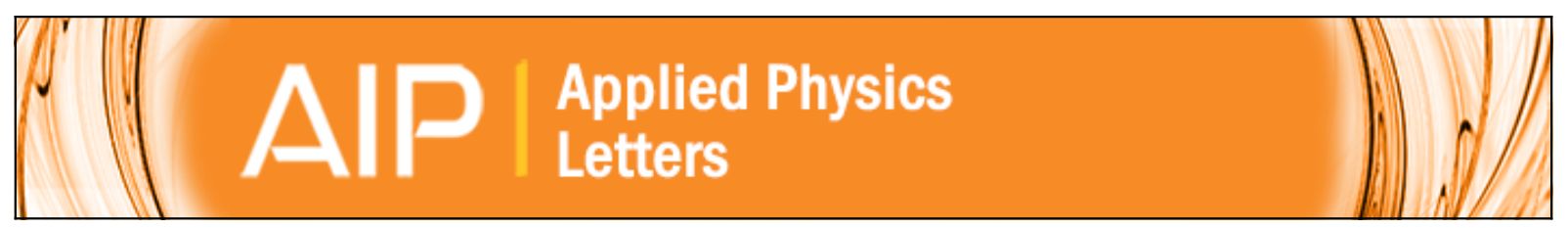

\section{Patterning of high mobility electron gases at complex oxide interfaces}

F. Trier, G. E. D. K. Prawiroatmodjo, M. von Soosten, D. V. Christensen, T. S. Jespersen, Y. Z. Chen, and N. Pryds

Citation: Applied Physics Letters 107, 191604 (2015); doi: 10.1063/1.4935553

View online: http://dx.doi.org/10.1063/1.4935553

View Table of Contents: http://scitation.aip.org/content/aip/journal/apl/107/19?ver=pdfcov

Published by the AIP Publishing

\section{Articles you may be interested in}

Growth-induced electron mobility enhancement at the LaAIO3/SrTiO3 interface

Appl. Phys. Lett. 106, 051604 (2015); 10.1063/1.4907676

Vacancy defect and carrier distributions in the high mobility electron gas formed at ion-irradiated SrTiO 3 surfaces

J. Appl. Phys. 107, 103704 (2010); 10.1063/1.3369438

Thickness dependence of the mobility at the LaAlO 3 / SrTiO 3 interface

Appl. Phys. Lett. 94, 222111 (2009); 10.1063/1.3149695

Microlithography of electron gases formed at interfaces in oxide heterostructures

Appl. Phys. Lett. 89, 122101 (2006); 10.1063/1.2354422

Extremely high-mobility two dimensional electron gas: Evaluation of scattering mechanisms

Appl. Phys. Lett. 71, 683 (1997); 10.1063/1.119829

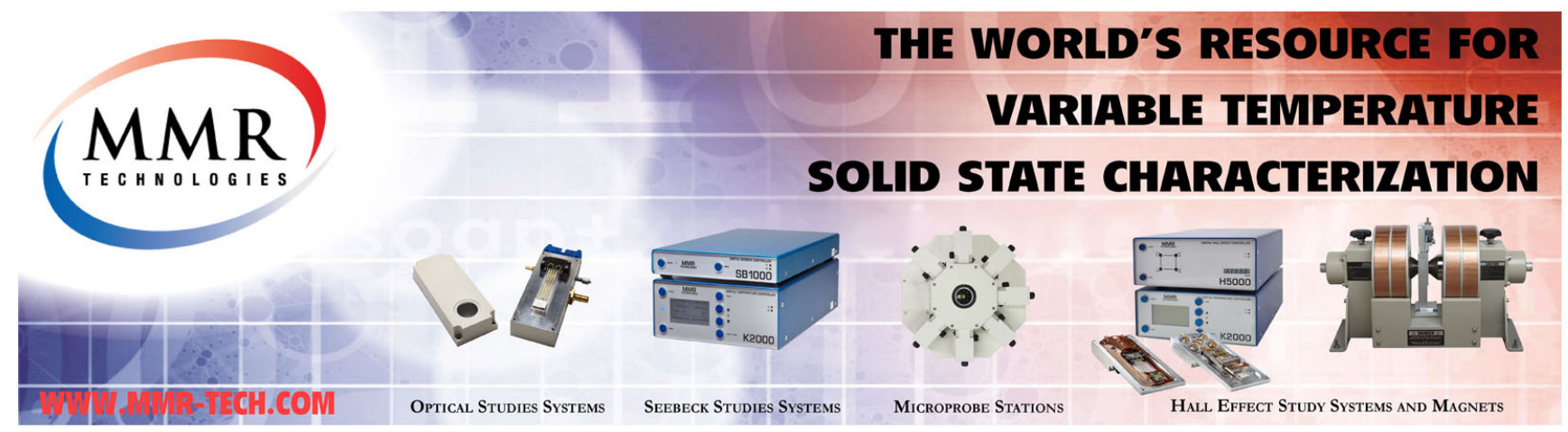




\title{
Patterning of high mobility electron gases at complex oxide interfaces
}

\author{
F. Trier, ${ }^{1, a)}$ G. E. D. K. Prawiroatmodjo, ${ }^{2}$ M. von Soosten, ${ }^{2}$ D. V. Christensen, ${ }^{1}$ \\ T. S. Jespersen, ${ }^{2}$ Y. Z. Chen, ${ }^{1}$ and N. Pryds ${ }^{1}$ \\ ${ }^{1}$ Department of Energy Conversion and Storage, Technical University of Denmark, Ris $\phi$ Campus, \\ DK-4000 Roskilde, Denmark \\ ${ }^{2}$ Center for Quantum Devices, Niels Bohr Institute, University of Copenhagen, DK-2100 Copenhagen $\emptyset$, \\ Denmark
}

(Received 5 October 2015; accepted 30 October 2015; published online 12 November 2015)

\begin{abstract}
Oxide interfaces provide an opportunity for electronics. However, patterning of electron gases at complex oxide interfaces is challenging. In particular, patterning of complex oxides while preserving a high electron mobility remains underexplored and inhibits the study of quantum mechanical effects where extended electron mean free paths are paramount. This letter presents an effective patterning strategy of both the amorphous-LaAlO $\mathrm{Al}_{3} / \mathrm{SrTiO}_{3}$ (a-LAO/STO) and modulation-doped amorphous- $\mathrm{LaAlO}_{3} / \mathrm{La}_{7 / 8} \mathrm{Sr}_{1 / 8} \mathrm{MnO}_{3} / \mathrm{SrTiO}_{3}$ (a-LAO/LSM/STO) oxide interfaces. Our patterning is based on selective wet etching of amorphous-LSM (a-LSM) thin films, which acts as a hard mask during subsequent depositions. Strikingly, the patterned modulation-doped interface shows electron mobilities up to $\sim 8700 \mathrm{~cm}^{2} / \mathrm{Vs}$ at $2 \mathrm{~K}$, which is among the highest reported values for patterned conducting complex oxide interfaces that usually are $\sim 1000 \mathrm{~cm}^{2} / \mathrm{V} \mathrm{s}$ at 2 K. (C) 2015 AIP Publishing LLC. [http://dx.doi.org/10.1063/1.4935553]
\end{abstract}

Research on interface phenomena between the two insulating oxides $\mathrm{LaAlO}_{3}$ (LAO) and $\mathrm{SrTiO}_{3}$ (STO) has resulted in the discovery of a wealth of attractive properties. These include a metallic two-dimensional electron gas (2-DEG), ${ }^{1}$ an electric field controlled metal-insulator transition, ${ }^{2,3}$ a superconducting phase ${ }^{4}$ tunable by application of an electric field, ${ }^{5,6}$ and ferromagnetic ordering. ${ }^{7}$ Herein, the charge carrier mobility represents the Achilles tendon of the interface conductivity as this generally tends to be low for complex oxides. Therefore, with the aim of improving the performance of oxide electronics ${ }^{8,9}$ and to allow the study of quantum mechanical effects where extended electron mean free paths are paramount, it is necessary to enhance the inherent charge carrier mobility. This has seen a significant progress with heterostructures of spinel structured $\gamma-\mathrm{Al}_{2} \mathrm{O}_{3}$ on STO (GAO/STO) displaying record-high electron mobilities of up to $140000 \mathrm{~cm}^{2} / \mathrm{V} \mathrm{s}$ at $2 \mathrm{~K} .{ }^{10}$ Recently, another high electron mobility system was discovered by introducing a single unit cell $\mathrm{La}_{7 / 8} \mathrm{Sr}_{1 / 8} \mathrm{MnO}_{3}$ (LSM) spacer layer between STO and amorphous-LAO grown at room temperature (RT). ${ }^{11}$ Strikingly, this system showed enhanced electron mobilities of up to $70000 \mathrm{~cm}^{2} / \mathrm{V} \mathrm{s}$ at $2 \mathrm{~K}$ compared to the usual $\sim 1000 \mathrm{~cm}^{2} / \mathrm{V} \mathrm{s}$ at $2 \mathrm{~K}$ for the a-LAO/ STO heterostructure. ${ }^{11,12}$

An equally important element inhibiting the advancement of oxide electronics and the ability to study quantum mechanical phenomena at mesoscopic scales is a lithographic patterning scheme which preserves the inherent interface quality of the system in spite of the processing, i.e., a strategy for patterning of the existing record-high mobility electron gases such as those found in GAO/STO or a-LAO/LSM/STO. Patterning of complex oxides has previously been addressed either relying on hard mask lift-off ${ }^{13-15}$ or low-energy ion

\footnotetext{
${ }^{\text {a) }}$ Author to whom correspondence should be addressed. Electronic mail: fetri@dtu.dk
}

beam irradiation. ${ }^{16}$ However, we found that these methods were challenging in patterning the high mobility GAO/STO heterostructure prepared at high temperature. ${ }^{10}$

Although high mobility oxide interfaces prepared at $\mathrm{RT}^{11,17}$ provides a straightforward way to pattern oxide interfaces with conventional lithography techniques utilizing, e.g., a resist soft mask, this results in insulating interfaces. Ultimately, state-of-the-art values of the electron mobility in patterned complex oxide interfaces typically remains around $3000 \mathrm{~cm}^{2} / \mathrm{V}$ s at $2 \mathrm{~K} .^{14,17}$

In this letter, we present a strategy which allows patterning of not only the a-LAO/STO interface conductivity but also the high mobility interface conductivity in the modulation-doped a-LAO/LSM/STO structure. To achieve this, we initially covered the bare STO surface with an amorphous-LSM (a-LSM) thin film grown at RT (see Fig. 1). Here, a-LSM is chosen primarily since a-LSM/STO heterostructures inherently are insulating ${ }^{12}$ regardless of temperature or oxygen partial pressure during the sample processing. During deposition of the LSM spacer layer $\left(\mathrm{P}_{\mathrm{O}_{2}} \approx 1 \times 10^{-4}\right.$, $\mathrm{T} \approx 600{ }^{\circ} \mathrm{C}$ ), the use of other potential hard mask materials such as amorphous aluminum oxide $\left(\mathrm{AlO}_{\mathrm{x}}\right)^{14}$ could be problematic as this may result in conducting $\mathrm{AlO}_{\mathrm{x}} / \mathrm{STO}$ interfa$\operatorname{ces}^{10,12,17}$ and thus prevent patterning of the interface conductivity. Furthermore, a-LSM is chosen since, by selective wet chemical etching, ${ }^{18}$ it can act as a hard shadow mask during the subsequent depositions. With a final deposition of a-LAO, this will result in conducting a-LAO/STO areas whereas a-LAO/a-LSM/STO regions remain insulating (see Fig. 1(f)) owing to their different redox-reactivity with the STO substrate. ${ }^{12}$ Remarkably, complex oxide Hall bar devices prepared with this patterning strategy show electron mobilities up to $\sim 8700 \mathrm{~cm}^{2} / \mathrm{V} \mathrm{s}$ at $2 \mathrm{~K}$.

Deposition of oxide thin films at RT permits the usage of soft resist masks, which otherwise would decompose at elevated deposition temperatures. However, directly 
e-beam resist

$\mathrm{SrTiO}_{3}(\mathrm{STO})$

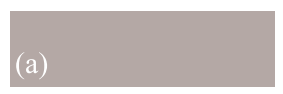

Bare STO substrate

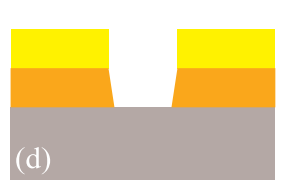

a-LSM KI/HCl etch
amorphous-LaSrMnO 3 (a-LSM)

amorphous- $\mathrm{LaAlO}_{3}$ (a-LAO)

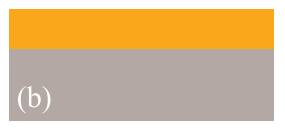

a-LSM deposition

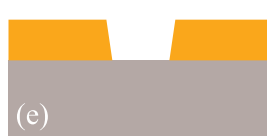

Resist removal and surface cleaning

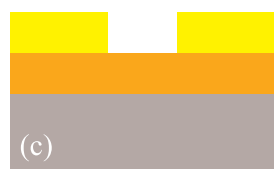

Resist exposure and development

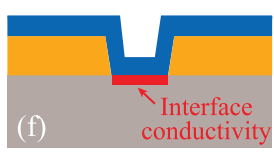

Deposition of either a-LAO or a-LAO/LSM

FIG. 1. Schematic illustration of the patterning process. (a) and (b) A bare $\mathrm{TiO}_{2}$-terminated $\mathrm{SrTiO}_{3}$ (STO) substrate is deposited with amorphous-La $\mathrm{La}_{7 / 8}$ $\mathrm{Sr}_{1 / 8} \mathrm{MnO}_{3}$ (a-LSM/STO). (c) The a-LSM/STO heterostructure is then prepared with an electron-beam (e-beam) defined resist pattern. (d) The sample is then subjected to selective $\mathrm{KI} / \mathrm{HCl}$ etching as directed by the resist. (e) The remaining resist is then removed and the sample surface is cleaned. (f) Deposition of amorphous- $\mathrm{LaAlO}_{3}(\mathrm{a}-\mathrm{LAO})$ results in either conducting a-LAO/STO or insulating a-LAO/a-LSM/STO heterostructures. The high electron mobility interface is obtained by deposition of a single unit cell LSM before the a-LAO deposition.

patterning the a-LAO/STO interface by use of a polymethyl methacrylate (PMMA) resist layer as a soft mask have been observed to result in insulating interfaces (data not shown). To prevent the resist layer from contaminating the delicate STO surface, we investigated a hard mask patterning strategy: the $\mathrm{TiO}_{2}$-terminated $\mathrm{STO}^{19}$ substrates are initially deposited with $60 \mathrm{~nm}$ of a-LSM using pulsed laser deposition (PLD) (see Fig. 1(b)). The a-LSM/STO samples are then prepared with a $200 \mathrm{~nm}$ thick PMMA electron-beam resist layer, which is exposed using a $100 \mathrm{kV}$ electron-beam into the desired Hall bar geometry (see Fig. 1(c)) and developed using 1:3 methyl isobutyl ketone and isopropanol (MIBK:IPA). To improve the resist adhesion with the a-LSM surface, the PMMA resist is reflown by post-baking at $185^{\circ} \mathrm{C}$ for $90 \mathrm{~s}$. The remaining resist will then protect underlying a-LSM from the etchant-a 2:2:35 $\mathrm{KI}(3 \mathrm{M}): \mathrm{HCl}(35 \%): \mathrm{H}_{2} \mathrm{O}$ acid solution. ${ }^{18}$ The a-LSM/ STO samples are etched for $15 \mathrm{~s}$ at a temperature of $20^{\circ} \mathrm{C}$ (see Fig. 1 (d)). This procedure allows pattern transfer with sub-micrometer resolution. To investigate the surface quality after etching, the sample surface at etched regions is probed using atomic force microscopy (AFM) (see Fig. 2(b)). For all measured samples, the STO surface structure is consistent with atomically flat $\mathrm{TiO}_{2}$ terraces that have a width similar to what is measured prior to the etching process. After the remaining resist is removed (see Fig. 1(e)), the structured aLSM/STO samples are transferred back to the PLD chamber where either a-LAO or a-LAO/LSM is deposited (see Fig. 1(f)). For the a-LAO/STO samples, a $16 \mathrm{~nm}$ a-LAO is deposited on the structured a-LSM/STO sample at RT with identical PLD parameters as previously reported. ${ }^{3,12}$ For the a-LAO/ LSM/STO samples, the structured a-LSM/STO sample is initially deposited with a single unit cell LSM spacer layer at $600^{\circ} \mathrm{C}$ and otherwise identical deposition parameters as recently reported. ${ }^{11}$ Subsequently, it is cooled under an oxygen pressure of $P_{\mathrm{O}_{2}} \approx 1 \times 10^{-4} \mathrm{mbar}$ with a rate of $15^{\circ} \mathrm{C} / \mathrm{min}$ to RT $\left(<25^{\circ} \mathrm{C}\right.$ ) followed by deposition of $16 \mathrm{~nm}$ a-LAO using

(a)

(b) Height $[\mathrm{nm}]$

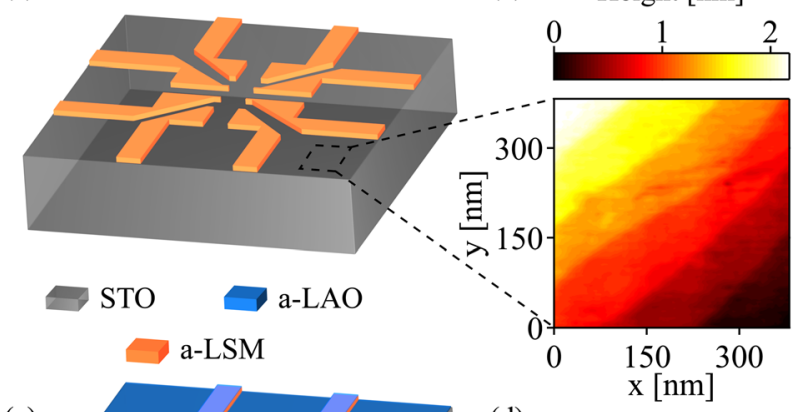

(d)
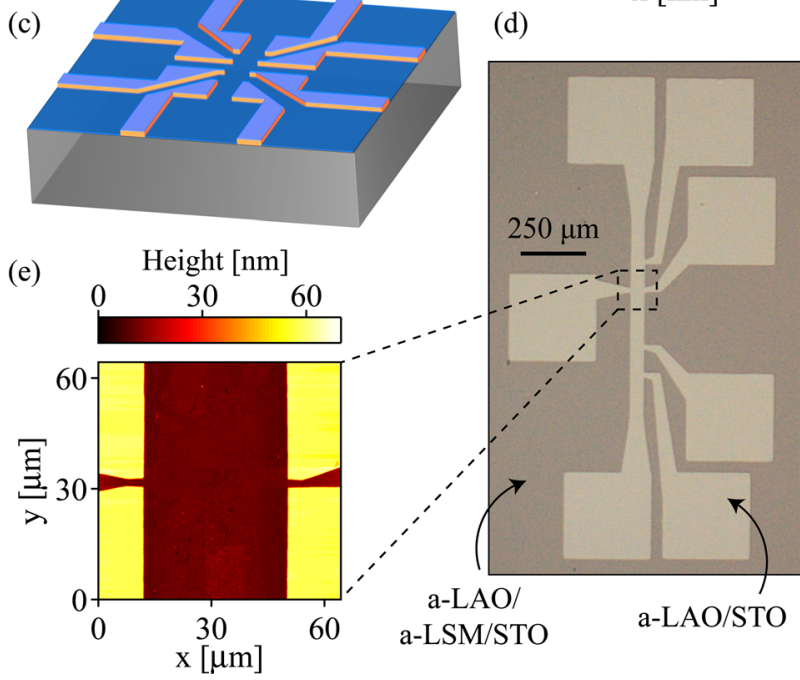

FIG. 2. (a) Schematic illustration of an etched a-LSM/STO heterostructure. The dashed square schematically represents the scanned area imaged by atomic force microscopy (AFM) in (b). (b) AFM image of a $\mathrm{KI} / \mathrm{HCl}$ etched region with visible STO terrace structure. (c) Schematic illustration of the patterned a-LAO/STO heterostructure. (d) Optical microscopy image of an a-LAO/STO Hall bar device with light and dark grey regions corresponding to areas with a-LAO/STO and a-LAO/a-LSM/STO, respectively. The dashed square indicates the AFM scanned area in (e). (e) AFM image of a Hall bar segment and two voltage probes, which shows sub-micrometer pattern-edge roughness.

the PLD parameters reported elsewhere. ${ }^{3,12}$ After final a-LAO deposition, the Hall bar devices are imaged using optical microscopy (see Fig. 2(d)), where light and dark grey regions correspond to areas with and without the a-LSM hard mask, respectively. This visible difference between the two areas allows for easy localization of the Hall bar devices. Additionally, Fig. 2(e) shows the device topography at two opposing voltage probes as imaged by AFM. The device topography is well defined and displays sub-micrometer patternedge roughness.

For comparison, unpatterned $5 \times 5 \mathrm{~mm}^{2}$ a-LAO/STO and a-LAO/LSM/STO reference samples (i.e., without a-LSM deposition or etching) are prepared and measured in the van der Pauw geometry. The interface of all samples is contacted using ultrasonically wire-bonded aluminum wires.

Fig. 3(a) shows the sheet resistance as a function of temperature for a representative a-LAO/STO Hall bar and the unpatterned a-LAO/STO reference sample. Both interfaces show comparable transport properties, indicating that the interface conduction generally is little affected by the patterning process. The a-LAO/STO Hall bar albeit displays a slightly higher sheet resistance than the unpatterned a-LAO/ STO at room temperature, but this difference diminishes as the temperature is decreased. This discrepancy is caused by 
(a)

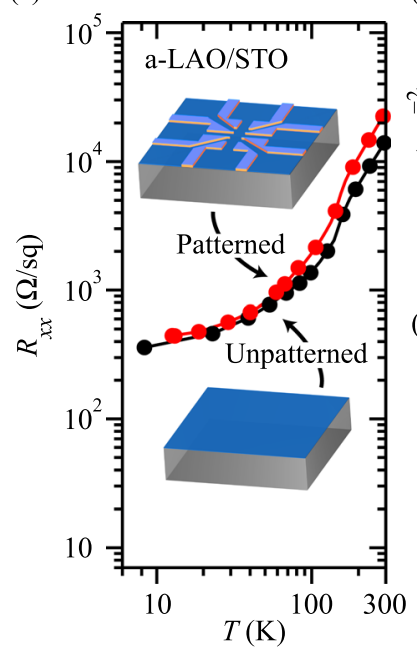

(b)

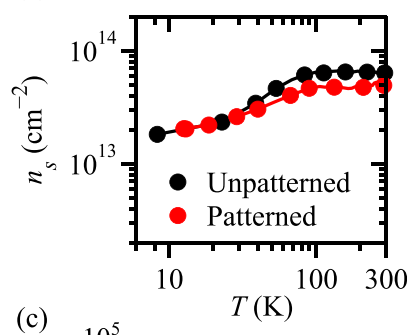

(c)

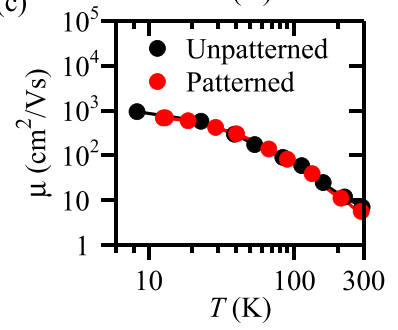

(a)

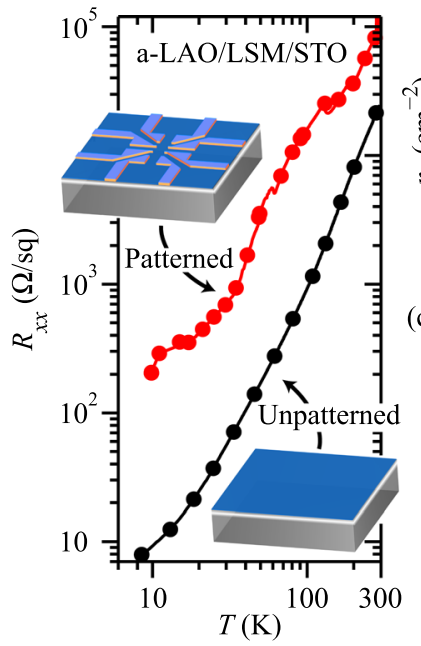

(b)

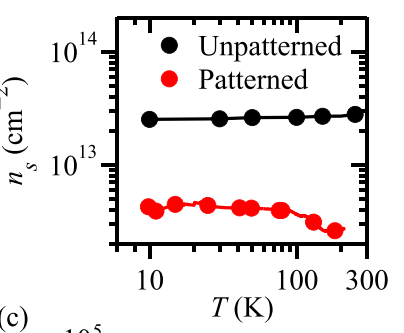

(c)

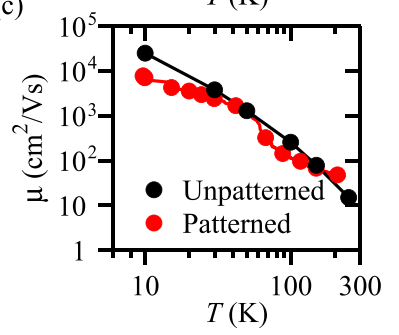

FIG. 3. (a) Sheet resistance $\left(R_{x x}\right)$ vs. temperature $(T)$ of a representative patterned a-LAO/STO Hall bar with a width of $50 \mu \mathrm{m}$ and a distance between longitudinal voltage probes of $300 \mu \mathrm{m}$ and the unpatterned a-LAO/STO reference sample, which indicates that the patterning process has little effect on the formation of interface conductivity. (b) Carrier density $\left(n_{s}\right)$ as a function of $T$ shows that there is a small difference between the two samples for $T>100 \mathrm{~K}$. (c) Comparing the electron mobility $(\mu)$ of the two samples shows that the quality of the interface is preserved in spite of the patterning process.

their minor carrier density difference for $T>100 \mathrm{~K}$ (see Fig. 3(b)) below which the cubic to tetragonal phase transition of STO occurs. ${ }^{20}$ Finally, as shown in Fig. 3(c), the two a-LAO/STO samples exhibit almost same electron mobilities, indicating that the quality/cleanness of the interface is preserved after the patterning process. Similarly, the mobility is comparable with typical values for patterned and unpatterned interface conductivity in most all-crystalline LAO/ STO heterostructures. ${ }^{13,15}$

Interestingly, this strategy is also applicable to pattern modulation-doped a-LAO/LSM/STO Hall bar devices with enlarged electron mobilities. As shown in Fig. 4, a patterned a-LAO/LSM/STO Hall bar device shows a carrier density of $5.6 \times 10^{12} \mathrm{~cm}^{-2}$ (see Fig. 4(b)), much lower than the a-LAO/ STO samples (see Fig. 3(b)). Moreover, the carrier density is little temperature dependent similarly to what is characteristic for the unpatterned a-LAO/LSM/STO heterostructure. Strikingly, the patterned a-LAO/LSM/STO Hall bar device shows a mobility of $8703 \mathrm{~cm}^{2} / \mathrm{Vs}$ at $2 \mathrm{~K}$ (see Fig. $4(\mathrm{c})$ ). Although this mobility remains almost an order below the record-high value of $70000 \mathrm{~cm}^{2} / \mathrm{V} \mathrm{s}$ for the unpatterned aLAO/LSM/STO reference sample, it is among the highest reported values for patterned complex oxide interfaces with the typical value for the patterned LAO/STO interface often being below $\sim 1000 \mathrm{~cm}^{2} / \mathrm{Vs}$ at $2 \mathrm{~K} .^{13,15}$ Such a high mobility and low carrier density interface in a-LAO/LSM/STO enables the observation of clear Shubnikov-de Haas oscillations and the initial manifestation of the quantum Hall effect in complex oxides. $^{11}$

To conclude, we outline a general strategy for patterning of metallic interfaces in complex oxide heterostructures prepared at RT. In particular, the strategy is based on selective etching of an a-LSM thin film acting as hard mask in subsequent film depositions. The technique is further found to be

FIG. 4. (a) $T$ dependence of $R_{x x}$ for a representative patterned a-LAO/LSM/ STO Hall bar with a width of $70 \mu \mathrm{m}$ and a distance between longitudinal voltage probes of $300 \mu \mathrm{m}$ and the unpatterned a-LAO/LSM/STO reference sample. (b) $n_{s}$ as a function of $T$ indicates a carrier density difference between the patterned and unpatterned samples which is consistent with their $R_{x x}$ discrepancy. (c) At low $T$, the electron mobility, $\mu$, of the two samples are consistent with high quality interfaces, and for the patterned sample, the measured $\mu$ is among the highest reported values for patterned complex oxide interfaces.

applicable for the modulation-doped oxide interface where the patterned interfaces show enhanced electron mobilities compared to typical values of the canonical LAO/STO interface. This opens the door to design oxide microelectronic devices and study mesoscopic physics based on complex oxides.

T.S.J. and G.E.D.K.P. acknowledge Lundbeck Foundation for financial support. The Center for Quantum Devices is supported by the Danish National Research Foundation.

${ }^{1}$ A. Ohtomo and H. Y. Hwang, Nature 427, 423-426 (2004).

${ }^{2}$ S. Thiel, G. Hammerl, A. Schmehl, C. W. Schneider, and J. Manhart, Science 313, 1942 (2006).

${ }^{3}$ D. V. Christensen, F. Trier, Y. Z. Chen, A. Smith, J. Nygård, and N. Pryds, Appl. Phys. Lett. 102, 021602 (2013).

${ }^{4}$ N. Reyren, S. Thiel, A. D. Caviglia, L. Fitting Kourkoutis, G. Hammer, C. Richter, C. W. Schneider, T. Kopp, A.-S. Rüetschi, D. Jaccard, M. Gabay, D. A. Muller, J.-M. Triscone, and J. Mannhart, Science 317, 1196 (2007).

${ }^{5}$ A. D. Caviglia, S. Gariglio, N. Reyren, D. Jaccard, T. Schneider, M. Gabay, S. Thiel, G. Hammerl, J. Mannhart, and J.-M. Triscone, Nature 456, 624 (2008).

${ }^{6}$ G. E. D. K. Prawiroatmodjo, F. Trier, D. V. Christensen, Y. Z. Chen, N. Pryds, and T. S. Jespersen, "Gate-tunable superconductivity at the amorphous $\mathrm{LaAlO}_{3} / \mathrm{SrTiO}_{3}$ interface," Phys. Rev. Lett. (submitted); preprint arXiv: 1510.01199.

${ }^{7}$ A. Brinkman, M. Huijben, M. van Zalk, J. Huijben, U. Zeitler, J. C. Maan, W. G. van der Wiel, G. Rijnders, D. H. A. Blank, and H. Hilgenkamp, Nat. Mater. 6, 493 (2007).

${ }^{8}$ D. G. Schlom and J. Mannhart, Nat. Mater. 10, 168-169 (2011).

${ }^{9}$ J. Mannhart and D. G. Schlom, Science 327, 1607 (2010).

${ }^{10}$ Y. Z. Chen, N. Bovet, F. Trier, D. V. Christensen, F. M. Qu, N. H. Andersen, T. Kasama, W. Zhang, R. Giraud, J. Dufouleur, T. S. Jespersen, J. R. Sun, A. Smith, J. Nygård, L. Lu, B. Büchner, B. G. Shen, S. Linderoth, and N. Pryds, Nat. Commun. 4, 1371 (2013).

${ }^{11}$ Y. Z. Chen, F. Trier, T. Wijnands, R. J. Green, N. Gauquelin, R. Egoavil, D. V. Christensen, G. Koster, M. Huijben, N. Bovet, S. Macke, F. He, R. Sutarto, N. H. Andersen, J. A. Sulpizio, M. Honig, G. E. D. K. Prawiroatmodjo, T. S. Jespersen, S. Linderoth, S. Ilani, J. Verbeeck, G. Van Tendeloo, G. Rijnders, G. A. Sawatzky, and N. Pryds, Nat. Mater. 14, 801 (2015). 
${ }^{12}$ Y. Z. Chen, N. Pryds, J. E. Kleibeuker, G. Koster, J. R. Sun, E. Stamate, B. G. Shen, G. Rijnders, and S. Linderoth, Nano Lett. 11, 3774 (2011).

${ }^{13}$ C. W. Schneider, S. Thiel, G. Hammerl, C. Richter, and J. Mannhart, Appl. Phys. Lett. 89, 122101 (2006).

${ }^{14}$ N. Banerjee, M. Huijben, G. Koster, and G. Rijnders, Appl. Phys. Lett. 100, 041601 (2012).

${ }^{15}$ D. Stornaiuolo, S. Gariglio, N. J. G. Couto, A. Fête, A. D. Caviglia, G. Seyfarth, D. Jaccard, A. F. Morpurgo, and J.-M. Triscone, Appl. Phys. Lett. 101, 222601 (2012).
${ }^{16}$ P. P. Aurino, A. Kalabukhov, N. Tuzla, E. Olsson, T. Claeson, and D. Winkler, Appl. Phys. Lett. 102, 201610 (2013).

${ }^{17}$ Y. Z. Chen, N. Bovet, T. Kasama, W. W. Gao, S. Yazdi, C. Ma, N. Pryds, and S. Linderoth, Adv. Mater. 26, 1462-1467 (2014).

${ }^{18}$ G. Bridoux, J. Barzola-Quiquia, F. Bern, W. Böhlmann, I. Vrejoiu, M. Ziese, and P. Esquinazi, Nanotechnology 23, 085302 (2012).

${ }^{19}$ M. Kareev, S. Prosandeev, J. Liu, C. Gan, A. Kareev, J. W. Freeland, Min Xiao, and J. Chakhalian, Appl. Phys. Lett. 93, 061909 (2008).

${ }^{20}$ F. W. Lytle, J. Appl. Phys. 35, 2212 (1964). 